\title{
Radionuclide X-ray fluorescence spectrometry of medical plant samples
}

\author{
Ol'ga Lukačovičová \\ Department of Pharmaceutical Analysis and Nuclear Pharmacy, Faculty of Pharmacy, \\ Comenius University in Bratislava, Odbojárov 10, 83232 Bratislava, Slovak Republic \\ lukacovicova@fpharm.uniba.sk
}

\begin{abstract}
The method of samples processing and subsequent their analysis using Radionuclide X-ray Fluorescence Spectrometry for medical plant samples and drugs has been developed. Technical aspects of the measurement such as mutual geometrical arrangement of tablets (of various size and mass), semiconductor $\mathrm{Si} / \mathrm{Li}$ detector and the primary source of radiation, ${ }^{238} \mathrm{Pu}$, are discussed. Technique of Radionuclide X-ray Fluorescence Spectrometry (RXFS) has been applied on samples of medical products and medicines used to treat liver diseases.

Medicinal herbs, teas in infusion bags and the drug from this group, were analysed in the solid state (in the form of tablets) or as the leachate, applied to the ion exchanger resin.

Chelating Extraction Disks Empore ${ }^{\mathrm{TM}}$ have proven the ability to extract elements from liquid samples under certain conditions and thus to prepare samples for RXFS analysis and evaluation. To ensure correct results, an optimal $\mathrm{pH}$ environment has been found. The detection limits of selected elements $(\mathrm{Cr}, \mathrm{Mn}, \mathrm{Fe}, \mathrm{Ni}, \mathrm{Cu}$, $\mathrm{Zn}$ and $\mathrm{Pb}$ ) have been calculated for both solid and liquid state samples. Standard addition method and calibration curve parameters have been used to evaluate the concentration of each element in studied samples. Determined concentrations of the examined elements were compared with permissible contaminant limits for the relevant food law in Slovak Republic.
\end{abstract}

Key words: Medical Plants; Liver Diseases; Radionuclide X-ray Fluorescence Spectrometry.

\section{Introduction}

Monitoring of quality control and evaluation of medicines and medicinal teas is an important aspect as in the production as well as in the distribution on the pharmaceutical market.

Preparations which are freely available to the patient may be at risk of exceeding the recommended dosage or dispensing the insufficient. As a result of the increased intake of medicinal products (though they are teas from medical plants), a large number of undesirable substances can accumulate in the body.

Pharmaceutical preparations - medicines, nutritional supplements or medicinal teas are produced by mass machine production. It can be assumed that contaminants (organic impurities, heavy metals) that are responsible for decreasing the quality of the preparations, are delivered during the work-up. Their undesirable accumulation in the body may give rise to a risk that outweighs the benefit of therapy (Afridi et al., 2011; Clark et al., 2007).

It is therefore necessary to check the quality of the preparation at each stage of manufacture before being placed on the market or even after the pharmaceutical preparations become available to patients. In order to ensure desired quality, it is necessary to develop a methodology for rapid and effective evaluation of pharmaceutical products. Despite many available analytical methods which are sensitive to low element concentrations, some of them often require complicated sample processing before analysis, or cannot detect a range of elements at once (Barin et al., 2016; Rudovica et al., 2014; Zaib et al., 2015; Kuśmierek et al., 2011).

Radionuclide X-ray Fluorescence Spectrometry (RXFS) proves to be an advantageous analytical method for polycomponent analysis with no material destruction, has been used for this purpose in many works (Dalipi et al., 2017; Sitko et al., 2015; Guerra et al., 2015; Bosco, 2013). Moreover, both solid and liquid samples can be analyzed.

The fluorescence energy values define directly the elements present in the analyzed sample - qualitative analysis. Radionuclide X-ray fluorescence spectrometry is applied to the quantitative analysis, using the dependence of the intensity of fluorescence emission on the content of the determined element.

With increasing content, the intensity of radiation also increases. Irradiation energy represents the qualitative parameter of the analyzed substance, while the intensity of radiation is a quantitative assessment of this element in the analyte.

To achieve good sensitivity, low detection limits and reliable results, it is important to ensure correct choice of a detection technique with appropriate type and energy of the exciting radiation source and by simultaneous eliminate of the absorption properties of the sample (for example by reducing its thickness). 
The sensitivity of the used method is $10^{-7} \mathrm{~g}$, given as the total content of the determined elements.

The choice of a radionuclide source is governed by the nature and composition of the sample. It is well known that the energy of the source has to be higher, than the absorption threshold of the analyzed element. RXFS allows determining of several elements at once, and hence it is a task to find a suitable source whose energy is higher than the energy of the last investigated element in the sample, i.e. the element with the highest Z (Lekki et al., 2017).

We have also addressed an issue to different techniques of sample preparation in the present article. To prepare the sample in a solid state, it must be homogenized and shaped so as to minimize the absorption of the primary and characteristic radiation in the matrix (in contrast to the saturated layer - the coarse layer). It is ideal to press tablets with a defined shape, so called "transition layer", or better in the form of a thin layer. When analyzing a very thin layer of the analyzed sample, the radiation absorption (both primary and characteristic) in the matrix is reduced as well as the backscatter. The intensity is directly proportional to the weight of the element being determined. The analytical curve, expressing the radiation intensity dependence on the weight of the element, has a linear course in the thin layer. In addition, the tablet parameters (weight and diameter) must be set with respect to the amount of analyzed sample. In this context the optimization of the geometric configuration of the measurement technique (mutual position of radiation source, samples and detector) has to be considered (Sun et al., 2015).

Analysis of liquids directly in the sampler causes radiation scattering and therefore it is appropriate to fix analysed material to a suitable carrier having the form of a thin layer.

The aim of the experiment is searching of a suitable carrier which ensures lower detection limits and reduced non-analytical signal. Besides, this thin layer should also provide next requirement such as a preconcentration of the examined analyte (Alvarez et al., 2004; Karapete et al., 2002; Hou et al., 2003; Pehlivan and Altun, 2007; Chatterjee et al., 2016).

Note that the chelating membrane used in our work for this purpose - Empore ${ }^{\mathrm{TM}}$ Extraction Disks with chelating resin, (based on Iminodiacetate functionalized Poly[styrenedivinylbenzene]), fulfills above mentioned important methodological criterions.

The presented paper describes the use of various techniques of the samples preparation, the choice of advantageous radiation source and the geometric arrangement of the equipment for analysis of medicinal products, medicinal teas and plants.

\section{Materials and Methods}

The preparations selected for evaluation and quality control are medicines and medicinal teas, suitable as prophylaxis or adjuvant therapy for diseases with liver disorders (Shaker et al., 2010; Capasso et al., 2000). Medicinal herbs Plantago Lanceolata L. (1), Galium Verum L. (2), Taraxacum officinalis L. (3), Schizandra chinensis Turcz (4), Silybum marianum L. (5) (Agrokarpaty s.r.o, Slovak Republic) and teas in infusion bags: Almaden (Urtica dioica L., Betula pendula Roth., Viola tricolor L., Fragaria vesca L; Herbata s.r.o. Czech Republic) (6), Teekanne liver and gallbladder (radix and folium of Taraxacum officinalis L., Mentha piperita L., Foeniculum vulgare Mill., Matricaria chamomilla L., Silybum marianum L.; Teekanne s.r.o. Czech Republic) (7) and the drug Lagosa (dry extract of Silybum marianum L.; Wörwag Pharma GmbH \& Co. KG, Germany) (8) were analysed in the solid state (in the form of tablets) or as the leachate, applicated on the ion exchanger resin.

Selected elements - chromium, manganese, iron, nickel, copper, zinc and lead have been identified and determined by nuclear analysis method of RXFS.

Analysis was carried out with a semiconductor detector $(\mathrm{Si} / \mathrm{Li})$ working at a voltage of $500 \mathrm{~V}$ for the energy area up to $30 \mathrm{keV}$ (manufactured by ÚJV Rež u Prahy, Czech Republic) - the detector was cooled in a liquid nitrogen atmosphere; evaluated through multichannel analyzer EG \& G ORTEC, model 919E Ethernim, with software MAESTRO for WINDOWS (version A65-B32, version 6.05). Measurement time was $2000 \mathrm{~s}$, samples were analyzed at ambient temperature.

At the beginning of the experiment, the energy calibration was performed using known standards of selected element, whereby the energy maxima in the measured spectra were assigned to the individual analysed elements. A radionuclide radiation source of ${ }^{238} \mathrm{Pu}$ was used as a source of primary radiation whose energy is higher than the absorption threshold of the last determined element in the analyzed matrix $\left({ }^{238} \mathrm{Pu}-\mathrm{a}\right.$ low energy gamma emitter, type Y1055, energy of 12-22 keV, a halflife of 86.4 years and an activity of $880 \mathrm{MBq}$, made by Amersham in the form of a flat disc source).

Used method is advantageous for the detection and determination of elements with an atomic number from 18 to 90 . Samples in solid form were dried, homogenized and compressed by a hand-held tablet press into tablets, of defined shape and weight.

In order to evaluate the selected elements in leachate from medical plants, the chelating membranes Empore $^{\mathrm{TM}}$ were used. Empore ${ }^{\mathrm{TM}}$ Extraction Disks with chelating resin consist of a polymer support (crosslinked poly[styrenedivinylbenzene] functionalized 
by bonding at the nitrogen atoms to iminodiacetic acid group (Varian, USA). The functional group exhibits differing ion exchange depending on the $\mathrm{pH}$ of a solution. Used iminodiacetic chelator has an affinity for multivalent transition elements. Ion exchange membranes are advantageous for analysis for their good handling, defined shape, size and thickness (a diameter of $47 \mathrm{~mm}$ and a thickness of $0.5 \mathrm{~mm})$.

Certified solutions were used to prepare standards by the standard addition method: $\mathrm{Cr}, \mathrm{Mn}, \mathrm{Fe}$, $\mathrm{Ni}, \mathrm{Cu}, \mathrm{Zn}, \mathrm{Pb}$ in the form of nitrates $-1.0 \mathrm{~g} / \mathrm{l}$ (Metrologický ústav Bratislava, Slovak Republic). Chemicals used for liquid extraction on the membrane: methanol, nitric acid (Merck, Darmstadt, Germany), ammonium acetate (Lachema, Brno, Czech Republic), deionizing water.

\section{Results and discussion}

To evaluate the technical aspects of accurate analysis, the following samples of medicinal plants and commercial teas were selected for the treatment of liver damage in one therapeutic group: medicinal herbs Plantago Lanceolata L. (1), Galium Verum L. (2), Taraxacum officinalis L. (3), Schizandra chinensis Turcz. (4), Silybum marianum L. (5) and teas in infusion bags: Almaden (6), Teekanne liver and gallbladder (7) and the drug Lagosa, (8).

Tablets of two different weights were used for the sample evaluation in solid state: a weight of approximately $0.3 \mathrm{~g}$, a diameter of $20 \mathrm{~mm}$ and, alternatively, tablets of smaller parameters, a weight of $0.1 \mathrm{~g}$, a diameter of $10 \mathrm{~mm}$. The thickness of both types amounted to $1 \mathrm{~mm}$, thus representing a transition layer.

Nevertheless, there was a need to address the current geometric configurations: the sample-sourcedetector arrangement. When small dimensional sources and low sample amounts are only available, a coaxial geometry must be used. All three objects then lie in one axis and do not shield the effective part of the detector window area.

In order to minimize the interfering background signal and to obtain maximum analytical information as well, reflective side geometry with a source located at the edge of the detector was used. This configuration ensure the maximum efficiency of the fluorescence radiation detection and reduces to a minimum the absorption of radiation of low energies in the space between the sample and the detector, as well as the radiation generated in the material of measuring head of the detector. Low energy radiation absorption in the surrounding air is also minimizes due small distance between sample and detector.

It was important to place the sample so as to increase the efficiency of registration of induced radiation.

Therefore, the sample tablet was situated in different positions, with respect to the detector window and the radiation source. The monitored measured signal is counted as the sum of the pulses in a defined interval symmetrically below the energy peak in the measured spectrum corresponding to the specific element in time. The highest measured counts, which were obtained after analysis, represent the best orientation of all monitored parameters such as the position of the sample towards to the radiation source and the detector window (angle between the sample and radionuclide source, distance of the sample from the detector window). The source was fixed at an angle of $45^{\circ}$ from the pad, to the edge of the detector window and the distance between centres of the source and the detector window was $15 \mathrm{~mm}$. A sample has been fixed at a height of $10 \mathrm{~mm}$ on the Myllar foil.

The optimization of the geometric arrangement was declared in evaluation of selected elements in the Plantago Lanceolata L. sample for tablets weighing of about $0.3 \mathrm{~g}$ and $20 \mathrm{~mm}$ in diameter, which were in different positions. The "A" position: $10 \mathrm{~mm}$ to the left from midpoint of center line between detector window and radionuclide source center; in the "B" position - the tablet lied directly in the middle between radionuclide source and detector window and in the "C" where, oppositely, the tablet was located $10 \mathrm{~mm}$ to the right (projected directly above the detector window). Table 1 shows measured count values which comprise non-analytical signals, too.

However, depending on the examined position, some of the analysed elements disappeared after

Tab. 1. Evaluation of optimal geometric layout: sample-source-detector; for tablets weighing 0.3 grams and a diameter of 20 millimeters of Plantago Lanceolata L. sample (impulses/2000s).

\begin{tabular}{lccccccccc}
\hline \multirow{2}{*}{$\begin{array}{l}\text { Sample } \\
\text { position }\end{array}$} & $\mathbf{K}$ & $\mathbf{C a}$ & $\mathbf{C r}$ & $\mathbf{M n}$ & $\mathbf{F e}$ & $\mathbf{N i}$ & $\mathbf{C u}$ & $\mathbf{Z n}$ \\
\cline { 2 - 9 } A & 4866 & 5021 & 280 & 415 & 1609 & 166 & 302 & 659 & 223 \\
$\mathbf{B}$ & $\mathbf{5 3 2 2}$ & $\mathbf{5 4 1 5}$ & $\mathbf{2 9 9}$ & $\mathbf{4 5 8}$ & $\mathbf{1 7 1 3}$ & $\mathbf{1 8 3}$ & $\mathbf{4 0 8}$ & $\mathbf{7 7 1}$ & $\mathbf{2 5 7}$ \\
C & 4977 & 5117 & 285 & 426 & 1647 & 175 & 329 & 711 \\
\hline
\end{tabular}


a background subtraction. Based on measured counts optimal sample position outcome to be "B". Therefore, all the samples of the given sizes were placed in this way.

Due to lack of material or difficulties with subsequent processing into a defined shape, the preparation of smaller tablets was necessary. Similarly, the same experiment was performed with samples elaborated into smaller tablet sizes (about $0.1 \mathrm{~g}$ and $10 \mathrm{~mm}$ in diameter). To be in conformity with previous measurements, analogous settings were examined.
However, at such tablet dimensions, angle between the fixed source and the sample and also the distance from the detector or radiation source changed after some adjustments as compared to former arrangement of the larger tablets. Results in Table 2 display that the variant " $\mathrm{C}$ " is the most suitable for this type of samples and above mentioned detector with defined parameters.

With the optimal geometric configuration in hand ("B" for larger size and "C" for smaller tablets), the individual plant samples and the Lagosa tablets were analysed (Fig. 1).

Tab. 2. Evaluation of optimal geometric layout: sample-source-detector; for tablets weighing 0.1 grams and a diameter of 10 millimetres of Plantago Lanceolata L. sample (impulses/2000s).

\begin{tabular}{|c|c|c|c|c|c|c|c|c|c|}
\hline \multirow{2}{*}{$\begin{array}{l}\text { Sample } \\
\text { position }\end{array}$} & \multicolumn{9}{|c|}{ Elements } \\
\hline & $\mathbf{K}$ & $\mathbf{C a}$ & $\mathbf{C r}$ & Mn & $\mathbf{F e}$ & $\mathbf{N i}$ & $\mathbf{C u}$ & $\mathbf{Z n}$ & $\mathbf{P b}$ \\
\hline A & 1591 & 1637 & 95 & 128 & 513 & 51 & 96 & 201 & 71 \\
\hline B & 1648 & 1716 & 99 & 146 & 526 & 59 & 111 & 242 & 84 \\
\hline C & 1782 & 1798 & 103 & 159 & 572 & 73 & 141 & 269 & 93 \\
\hline
\end{tabular}

a)

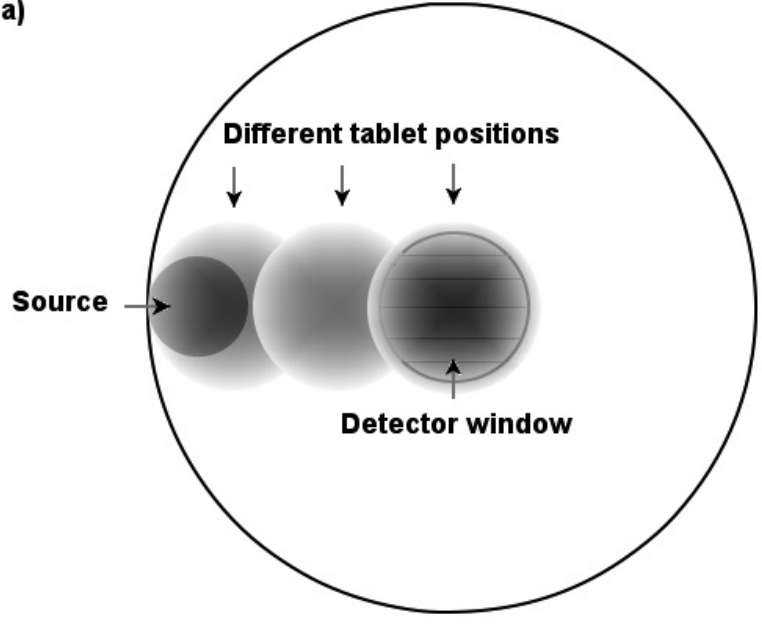

b)

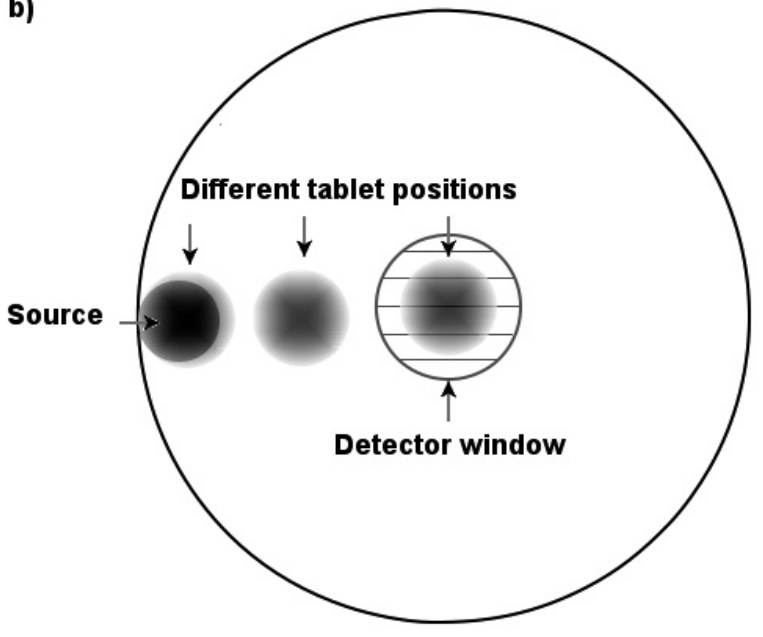

c)

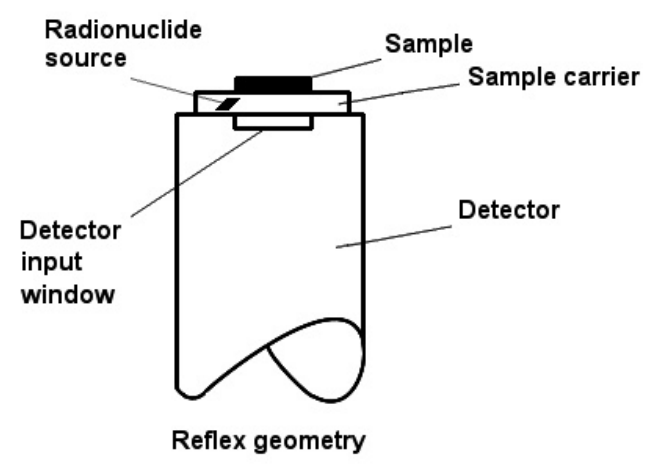

Notation

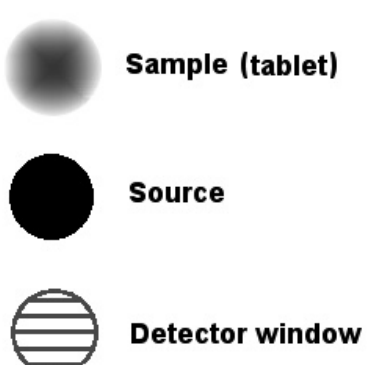

Fig. 1. Geometrical arrangement of the experiments (tablets position - upper projection a and b; c - lateral projection). 
In the measured spectra the present elements were identified via energy corresponding to the peak maximum. Figures 2 and 3 show radiation spectra after interaction of radionuclide source ${ }^{238} \mathrm{Pu}$ with the sample. The figures indicate energy maxima, through which the present elements were identified.

For determination of corresponding concentration of selected elements adequate standards were prepared by the method of standard addition. Moreover, it was important that the prepared standard for calculation of the elements concentration in the samples have the same real background as the plant samples and also have a similar elemental composition as the samples. Standard 1 was prepared by application $25 \mu \mathrm{g} / \mathrm{g}$ for $\mathrm{Cr}, \mathrm{Mn}, \mathrm{Ni}, \mathrm{Pb}$ and $50 \mu \mathrm{g} / \mathrm{g}$ for $\mathrm{Fe}, \mathrm{Cu}, \mathrm{Zn}$. Standard 2 differed from standard 1 in Fe and $\mathrm{Zn}(100 \mu \mathrm{g} / \mathrm{g})$ applied directly to the original matrix.

As a result, a variety of standards with different combinations of standard additives was prepared. Next variant of evaluation relied on construction of calibration lines (dependence of measured counts on the concentration) which also confirmed the linearity of the measured signal versus element addition in the range of $0-200 \mathrm{mg} / \mathrm{kg}$ (the calculated

Plantago lanceolata, $\mathrm{L} .+$ Standards

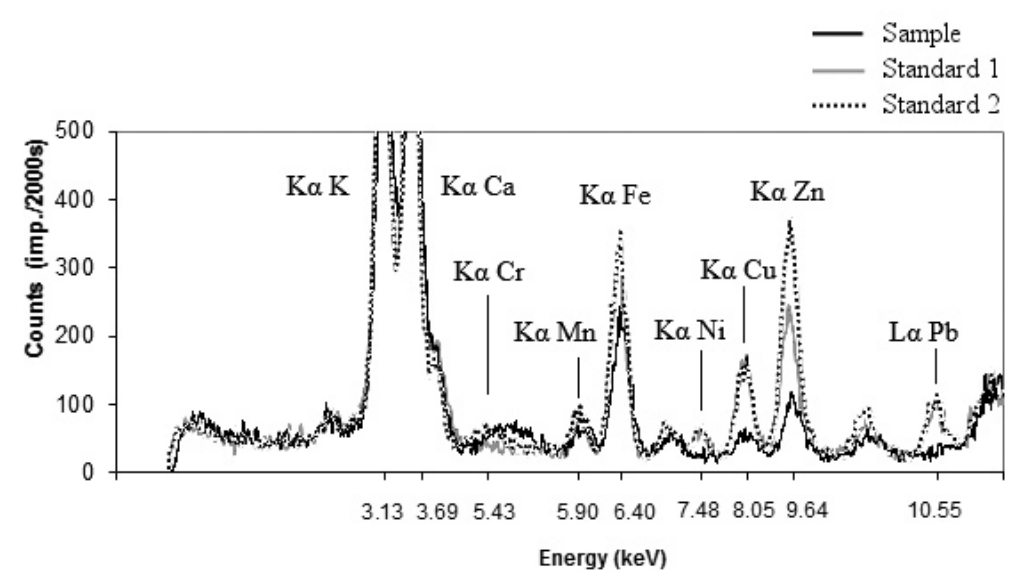

Fig. 2. XRF spectrum of Plantago lanceolata, L. sample and two different standard.

Tab. 3. The concentration of selected elements in the samples - different tablets parameters $(\mathrm{mg} / \mathrm{kg})$.

\begin{tabular}{|c|c|c|c|c|c|c|}
\hline \multirow{2}{*}{ Sample } & \multicolumn{6}{|c|}{ Elements } \\
\hline & $\mathrm{Cr}$ & Mn & $\mathrm{Fe}$ & $\mathbf{C u}$ & $\mathrm{Zn}$ & $\mathbf{P b}$ \\
\hline $1 \mathrm{a}$ & $<\mathrm{L}_{\mathrm{D}}$ & 21.83 & 122.08 & 5.11 & 21.18 & $<\mathrm{L}_{\mathrm{D}}$ \\
\hline $1 b$ & $<\mathrm{L}_{\mathrm{D}}$ & 21.27 & 121.66 & 5.03 & 20.56 & $<\mathrm{L}_{\mathrm{D}}$ \\
\hline $2 \mathrm{a}$ & 2.87 & 46.35 & 139.09 & 7.56 & 43.92 & $<\mathrm{L}_{\mathrm{D}}$ \\
\hline $2 b$ & 2.46 & 45.65 & 136.82 & 7.19 & 42.67 & $<\mathrm{L}_{\mathrm{D}}$ \\
\hline $3 a$ & $<\mathrm{L}_{\mathrm{D}}$ & 37.66 & 55.62 & 14.32 & 14.74 & $<\mathrm{L}_{\mathrm{D}}$ \\
\hline $3 \mathrm{~b}$ & $<\mathrm{L}_{\mathrm{D}}$ & 37.09 & 54.56 & 14.10 & 14.46 & $<\mathrm{L}_{\mathrm{D}}$ \\
\hline $4 \mathrm{a}$ & $<\mathrm{L}_{\mathrm{D}}$ & 50.83 & 53.78 & 11.89 & 10.21 & 2.15 \\
\hline $4 \mathrm{~b}$ & $<\mathrm{L}_{\mathrm{D}}$ & 49.51 & 51.84 & 11.55 & 9.82 & 2.08 \\
\hline $5 a$ & $<\mathrm{L}_{\mathrm{D}}$ & 16.07 & 55.15 & 24.35 & 87.91 & $<\mathrm{L}_{\mathrm{D}}$ \\
\hline $5 \mathrm{~b}$ & $<\mathrm{L}_{\mathrm{D}}$ & 15.97 & 53.84 & 24.26 & 86.64 & $<\mathrm{L}_{\mathrm{D}}$ \\
\hline $6 a$ & $<\mathrm{L}_{\mathrm{D}}$ & 300.81 & 56.22 & 5.81 & 62.09 & $<\mathrm{L}_{\mathrm{D}}$ \\
\hline $6 \mathrm{~b}$ & $<\mathrm{L}_{\mathrm{D}}$ & 302.86 & 55.16 & 5.63 & 61.20 & $<\mathrm{L}_{\mathrm{D}}$ \\
\hline $7 \mathrm{a}$ & $<\mathrm{L}_{\mathrm{D}}$ & 43.66 & 249.67 & 4.68 & 26.85 & $<\mathrm{L}_{\mathrm{D}}$ \\
\hline $7 \mathrm{~b}$ & $<\mathrm{L}_{\mathrm{D}}$ & 42.95 & 248.87 & 4.54 & 25.97 & $<\mathrm{L}_{\mathrm{D}}$ \\
\hline $8 \mathrm{a}$ & $<\mathrm{L}_{\mathrm{D}}$ & 12.11 & 130.54 & 2.81 & 2.14 & $<\mathrm{L}_{\mathrm{D}}$ \\
\hline $8 \mathrm{~b}$ & $<\mathrm{L}_{\mathrm{D}}$ & 11.84 & 127.76 & 2.80 & 2.11 & $<\mathrm{L}_{\mathrm{D}}$ \\
\hline
\end{tabular}

a: the samples prepared in form of tablets with a mass of $0.3 \mathrm{~g}$ and a diameter of $20 \mathrm{~mm}$. b: the samples prepared in form of tablets with a mass of $0.1 \mathrm{~g}$ and a diameter of $10 \mathrm{~mm}$. $\mathrm{L}_{\mathrm{D}}$ : detection limit. 
correlation coefficients were close to 1 ). Based on the line parameters the element concentration in the samples was calculated.

Drug Lagosa did not have the character of a biological sample and therefore benzoic acid that met the requirements for this type of standard was employed to produce an appropriate standard. The tablets of the standards were prepared using the same procedure as the samples.

The results of the calculated concentrations of analysed elements for both types of prepared tablets are given in Table 3. In the following text are listed some limits for permissible contaminants, which include the relevant laws in Slovakia: for chrome $1.0 \mathrm{mg} / \mathrm{kg}$ for beverages and $4.0 \mathrm{mg} / \mathrm{kg}$ for other foods, for lead $10 \mathrm{mg} / \mathrm{kg}$ for beverages and $1 \mathrm{mg} / \mathrm{kg}$ for other foods, for copper $150.0 \mathrm{mg} / \mathrm{kg}$ for beverages for tea and $25.0 \mathrm{mg} / \mathrm{kg}$ for other foods, for nickel $6.0 \mathrm{mg} / \mathrm{kg}$ for nutritional supplements (Ministry of Agriculture, 2004); the quantities of contaminants for iron are $15.0 \mathrm{mg} / \mathrm{kg}$ for beverages and unlimited for other foods and for zinc $80 \mathrm{mg} / \mathrm{kg}$ for other foods (Ministry of Health, 1994). Nickel in all our samples was under the detection limit. The standard deviation calculated from the variation range, and the relative standard deviation for each experiment was not exceeded (not more than $3 \%$ ).

Percentual differences of calculated element concentrations in analysed samples for various type of tablets were in the range between 0.35-5.14.

The following detection limits for solid state samples were calculated from non-analytical signal and determined parameters of the calibration curves or from the results of the evaluated standards, respectively: for Cr 2.28; Mn: 2.85; Fe: 2.64; Ni: 2.04; Cu: 2.35; Zn: 2.27; Pb: 1.98 (mg/kg).

Another problem to be solved was the treatment of liquid samples. Water is a strategic raw material, it is a component part of medicines and drugs. The quality of the prepared leachate from medical herbs depends thereby also on its quality.

The technique of sample preparation reflects type of analysed material considering quantity and character of impurities. From different approaches in the case of aqueous samples - extraction, lyophilization, filtration, precipitation, etc. (Berglund et al., 2013 Vuckovic, 2012, Alvarez-Sanchez et al., 2010), the ion exchange membrane technique seems to be the preconcentration method with many advantages (eliminated complicated chemical operations and does not cause additional contamination).

Chelating resin disks represent a tool to selectively remove multivalent metal cations from aqueous solutions by simply passing the solution through the porous membrane. Iminodiacetic chelator has an affinity for multivalent (primarily $2+$ and $3+$ ) transition elements. The binding of individual elements depends on the $\mathrm{pH}$ of the medium.

The membranes were placed on the basis of a vacuum apparatus and, according to the manufacturer's recommendation, first moistened with methanol, to swell the sorbent, then conditioned with nitric acid and converted to ammonium form with ammonium acetate.

Next, the constant volume of the sample was filtered through the chelation disc by suction with the vacuum apparatus.

Prior to further analysis the used disk with metal ions intercepted on the sorbent must be eluted first with 3 molar nitric acid, then washed with deionized water and converted finally to ammonium form with $100 \mathrm{mM}$ ammonium acetate buffer at pH 5.3.

In order to find appropriate $\mathrm{pH}$ conditions for the sorption of individual elements, liquid media of pH 3, pH 5 and pH 9 were prepared and tested.

Chelating Extraction Disks Empore ${ }^{\mathrm{TM}}$ meet thin film conditions $(0.5 \mathrm{~mm}$ thick) required for Radionuclide X-ray Fluorescence Spectrometry as mentioned above. Its diameter is $47 \mathrm{~mm}$, it fills the entire surface of the Myllar foil holder and thus eliminates the problem with position of the sample with respect to the detector and the radiation source.

Table 4 shows the measured counts from the extraction of the various elements $(50 \mathrm{mg} / \mathrm{kg})$ in volume $250 \mathrm{~mL}$ of the media $\mathrm{pH} 3, \mathrm{pH} 5$ and $\mathrm{pH} 9$ (standards for liquid samples)

The results exhibit that the most suitable medium for element analysis using chelating Extraction Disks Empore ${ }^{\mathrm{TM}}$ proves to be $\mathrm{pH}$ 9. It is clearly seen from the Figure 3 that at $\mathrm{pH} 3$ the elements of the standard sample were not captured or only to a very small extent; but at pH 9 significant peaks of captured elements are displayed.

Tab. 4. Measured counts (impulses/2000s) for selected elements (50 mg/kg) in $250 \mathrm{~mL}$ media of various $\mathrm{pH}$.

\begin{tabular}{lccccccc}
\hline \multirow{2}{*}{ pH } & $\mathbf{9}$ & $\mathbf{9}$ Elements \\
\cline { 2 - 9 } & Cr & Mn & Fe & Ni & Cu & Zn & Pb \\
\hline pH 3 & 379 & 426 & 447 & 387 & 474 & 491 & 412 \\
pH 5 & 289 & 322 & 365 & 323 & 338 & 376 & 319 \\
pH 9 & $\mathbf{5 5 9}$ & $\mathbf{6 8 3}$ & $\mathbf{8 8 3}$ & $\mathbf{1 0 1 6}$ & $\mathbf{1 3 7 2}$ & $\mathbf{1 7 0 7}$ & $\mathbf{1 2 5 8}$ \\
\hline
\end{tabular}




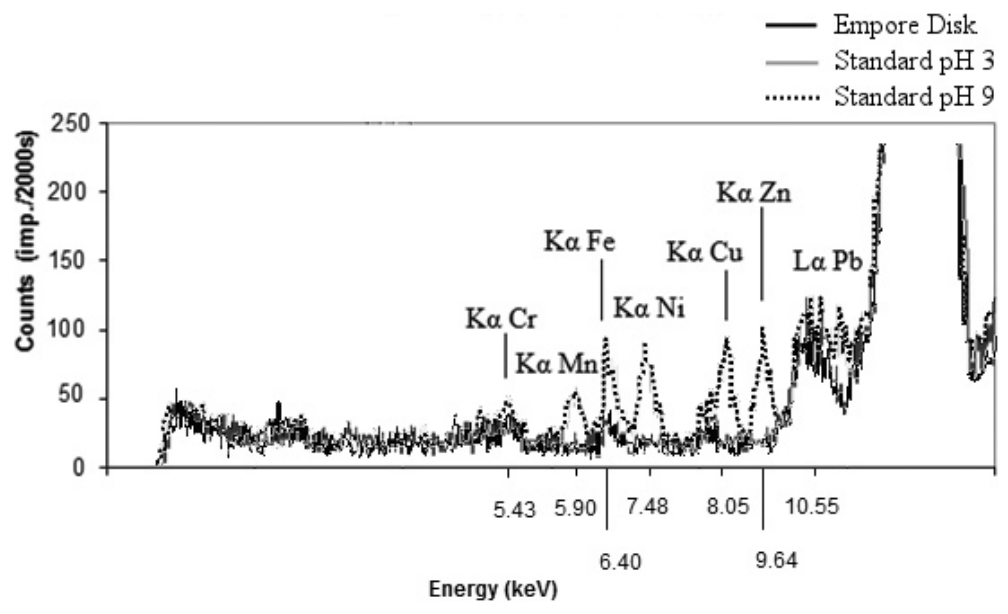

Fig. 3. XRF spectrum of the sample of conditioned chelating Extraction Disks Empore ${ }^{\mathrm{TM}}$ and standards prepared by adding of selected elements into $250 \mathrm{~mL}$ of deionized water adjusted to pH 3 and pH 9.

Consequently, prepared leachates from medical plants were adjusted to the appropriate $\mathrm{pH}$ and extracted through chelating membranes, then analysed and evaluated using the calibration curve parameters for the individual elements in the given medium.

Calculated detection limits for the method using a sample in a thin layer $(\mathrm{mg} / \mathrm{kg})$ : Cr 1.97; Mn: 2.05; Fe: 1.95; Ni: 1.51; Cu: 1.78; Zn: 1.87; Pb: 1.23.

The solution after the extraction of the sample was passed again through another membrane to see if all the presented elements were captured without the rest or if the capacity of the disk was not exceeded. Indeed, after the re-extraction of the solution from the previous operation it was found that leachates did not show significant values of the element concentrations. Subsequently, the dry residue of the plant material after the leaching was analysed to verify presence of the elements in the original plant material; it means they pass into the leachate only in limited quantity.

It turned out that significant levels of analyzed elements were remained in the original plant.

Clearly, these findings manifest that Radionuclide X-ray Fluorescence Spectrometry is effective quantitative analytical method for evaluation of easily extractable material or a soluble substance, advantageously at $\mathrm{pH} 9$.

\section{Conclusion}

In this work, Radionuclide X-ray Fluorescence Spectrometry was used for the evaluation of plant materials and medicines with the therapeutic effect intended to treat liver diseases.

Apparently, presented results show that accuracy and precision of RXRF measurements depend on the preparation of the analysed samples, on the technical aspects of the instrumentation, their settings, etc.

Depending on the quantity and availability of the material, as well as the property of the well-processed material, it is possible to choose the shape of the tablets for analysis.

Each prepared tablet type (different mass and diameter) requires the use of an optimal, characteristic geometric arrangement of the instrument system.

For tablets of smaller dimensions, it is a coordinate - coaxial of geometry, and for larger tablets a lateral geometry, while maintaining the radiation source at the edge of the detector window.

After identification of selected elements in the measured spectrum, a series of standards and calibration curves are required for quantification. The best way is to prepare them from original materials. For problematic samples, it is necessary to seek adequate substitutes for the preparation of the standard.

The experiment has shown that the use of different types of tablets does not affect the final concentration of the present elements, while preserving the specific geometric configuration of the instrumentation.

When analyzing liquid samples, it is an advantage to employ Empore ${ }^{\mathrm{TM}}$ chelating membranes which have affinity for the analysed elements with respect to the $\mathrm{pH}$ environment in which they are located.

It was confirmed that the thickness of the sample in this nuclear analytical method has important significance. This can be seen on the calculated detection limits that are higher for the transition layer (with thickness app. $1 \mathrm{~mm}$ ) used for tablets. However, for the thin layer (thickness app. $0.1 \mathrm{~mm}$ ) representing the chelating disk they are significantly lower. 
In the case of a soluble substance, it is recommended to use the observed $\mathrm{pH} 9$.

The detection limits of the method for the selected elements are below the limits stated in the relevant food laws in Slovak Republic. The results of the concentrations of the elements of the analysed samples also meet these limits, except iron. However, it is unlimited for category "other foods". Likewise, nickel can be evaluated in preparations as "other foods". Neither nickel, nor chromium did not exceed even the detection limits in the samples.

Remarkably, chromium concentration twice as high as permitted by the relevant law (the maximum permissible quantity of contaminants in foodstuffs valid in the Slovak Republic) was determined in the Galium verum $\mathrm{L}$., also exceeds the limits in the Schizandra chinensis Turcz. sample for lead concentration.

This method has the advantage that it is nondestructive, polycomponent, fast, and is suitable for analyzing different kinds of biological samples in both solid and liquid states.

\section{Acknowledgment}

The realisation of the experiment was supported by Slovak Grant Agency VEGA 1/0463/18, Bratislava, Slovak Republic.

\section{References}

Afridi HI, Kazi TG, Kazi N, Kandhro GA, Baig JA, Jamali MK, Arain MB, Shah AQ, Khan S, Kolachi NF (2011) Biol. Trace Elem. Res. 141: 26-40.

Álvarez-Sánchez B, Priego-Capote F, Luque de Castro MD (2010) Trends Anal. Chem. 29: 120-127.

Alvarez MB, Malla ME, Batistoni DA (2004) Anal. Bioanal. Chem. 378: 438-446.

Barin JS, Mello PA, Mesko MF, Duarte FA, Flores EMM (2016) Anal. Bioanal. Chem. 408: 4547-4566.

Berglund EC, Kuklinski NJ, Karagündüz E, Ucar K, Hanrieder J, Ewing AG (2013) Anal. Chem. 85: 2841-2846.
Bosco GL (2013) Trends Anal. Chem 45, 121-134.

Capasso R, Izzo AA, Pinto L, Bifulco T, Vitobello C, Mascolo N (2000) Fitoterapia 71: 58-65.

Clark NA, Teschke K, Rideout K, Copes R (2007) Chemosphere 70: 155-164.

Dalipi R, Marguí E, Borgese L, Depero LE (2017) Food Chem. 218: 348-355.

Guerra MBB, Adame A, Almeida E, Carvalho GGA, Brasil MAS, Santos Jr D, Krug FJ (2015) J. Anal. At. Spectrom. 7: 1646-1654.

Hou XD, Peters HL, Yang Z, Wagner KA, Batchelor JD, Daniel MM, Jones BT (2003) Appl. Spectrosc. 57: 338-342.

Chatterjee M, Srivastava B, Barman MK, Mandal B (2016) J Chromatogr., A 1440: 1-14.

Karapete AU, Soylak M, Elci L (2002) Anal. Lett. 35: $1561-1574$.

Kuśmierek K, Chwatko G, Głowack R, Kubalczyk P, Blad E (2011) J. Chromatogr., B: Anal. Technol. Biomed. Life Sci. 879: 1290-1307.

Lekki J, Matosz M, Paluszkiewicz C, Pieta E, Pieprzyca T, Szklarz Z, Meléndez JMH (2017) J. Radioanal. Nucl. Chem 314/3: 2309-2316.

Pehlivan E, Altun T (2007) J Hazard Mater 140: 299-307.

Rudovica V, Viksna A, Actins AJ (2014) Pharm. Biomed. Anal. 91: 119-122.

Sitko R, Janik P, Zawisza B, Talik E, Margui E, Queralt I (2015) Anal. Chem. 87: 3535-3542.

Shaker E, Mahmoud H, Mnaa S (2010) Food Chem. Toxicol. 48: 803-806.

Sun Y, Gleber SCh, Jacobsen Ch, Kirz J, Vogt S (2015) Ultramicroscopy 152: 44-56.

Vuckovic D (2012) Anal. Bioanal. Chem. 403: 1523-1548.

Zaib M, Athar MM, Saeed A, Farooq U (2015) Biosens. Bioelectron. 74: 895-908.

Food Codex Decree of Ministry of Agriculture Decree of Slovak Republic and Ministry of Health of Slovak Republic of 15 March 2004 no. 328/2004-100 and no. 608/3/2004-100 (Annex 2 to Section X of Part II of the Food Code, Part A - Chemicals) and Annex to Decree No. 18558/2006, the maximum permissible quantity of contaminants in foodstuffs valid in the Slovak Republic.

Ministry of Health Decree of Slovak Republic 2/1994 (contaminants - Chemical elements). 